\title{
Adrenal Insufficiency: An Uncommon Cause of Fatigue
}

\author{
Mitchell S. King, MD
}

Background: Adrenal insufficiency is a rare condition that can cause common and nonspecific symptoms. One such symptom, reported by all patients with adrenal insufficiency, is fatigue. On the other hand, up to $20 \%$ of patients seeking care from primary care physicians will have fatigue as a complaint. Only a small percentage of patients are found to have underlying medical disease.

Methods: A MEDLINE literature search was performed from 1966 to the present using the key words "fatigue," "adrenal insufficiency," and "polyglandular autoimmune endocrinopathy." Major endocrinology textbooks were also referenced. In addition, references were obtained from bibliographies of available articles.

Results and Conclusions: This article describes a patient with adrenal insufficiency and fatigue as the primary complaint. A brief discussion of fatigue and clues to organic causes follows, along with a more detailed discussion of adrenal insufficiency. Important medical history or signs and symptoms of organic disease suggest the need for screening tests and more detailed evaluation to uncover the uncommon medical causes of fatigue. (J Am Board Fam Pract 1999;12:386-90.)

Addison disease, or primary adrenal insufficiency, is a rare and potentially life-threatening disease. The prevalence of Addison disease is between 40 and 90 per 1 million persons. ${ }^{1,2}$ Patients with Addison disease often have nonspecific complaints that can easily be attributed to other causes. Nearly all patients with Addison disease have fatigue, and many have it as their primary complaint. ${ }^{3}$ Defined as tiredness or diminished energy level to the point of interfering with normal or usual activities, fatigue is a common symptom, reported by more than $20 \%$ of patients coming to primary care physicians. ${ }^{4}$ In a population survey, fatigue for longer than 6 months was reported by $19 \%$ of the respondents. ${ }^{5}$ Determining who among the many patients with fatigue might have an underlying medical disease is a challenging problem. The following case describes a patient with Addison disease, as part of Schmidt syndrome, whose chief complaint was fatigue. A discussion of fatigue and adrenal insufficiency and brief review of the literature provide insight into the challenges of diagnosing rare medical conditions in patients with common complaints.

Submitted, revised, 5 September 1998.

From the Northwestern University Medical School, Chicago. Reprints are not available.

\section{Methods}

A MEDLINE literature search was undertaken using the key words "fatigue," "adrenal insufficiency," and "polyglandular autoimmune endocrinopathy" for the years 1966 to the present. Major endocrinology textbooks were also referenced. In addition, references were obtained by using the bibliographies of available articles.

\section{Case Report}

A 48 year-old married woman came to the Family Practice Center complaining of a 1-year history of increasing fatigue and dull occipital headaches without any neurologic complaints. She denied fever, nausea, vomiting, or history of trauma. She did complain of hot flashes and oligomenorrhea for which she was seeing her gynecologist. The headache symptoms troubled the patient because her mother died of a brain tumor at 52 years. The patient's fatigue persisted throughout the day, worsened with activity, and caused her to feel depressed.

Her medical history was noteworthy for Hashimoto thyroiditis, for which she was taking levothyroxine $0.15 \mathrm{mg}$ daily. Her other medications included calcium citrate $600 \mathrm{mg}$ twice a day, a multivitamin daily, and aspirin $325 \mathrm{mg}$ every other 
day. In addition to her mother's brain tumor (unknown type), she had an aunt with type 2 diabetes mellitus. The patient was a homemaker who had 2 children for whom "she lived for" and a "very functional" chronic alcoholic husband. She denied alcohol or tobacco use, and had stopped exercising 1 year ago because of the fatigue. During a review of systems, some slight thinning of her scalp hair was noted, and she reported normal laboratory findings during a gynecology visit 2 months earlier.

When examined, her temperature was $97.4^{\circ} \mathrm{F}$, her blood pressure was $90 / 60 \mathrm{~mm} \mathrm{Hg}$, her heart rate was 88 beats per minute, and her respiratory was rate $16 / \mathrm{min}$. Findings during the remainder of the physical examination were normal. The initial impression was that the patient's symptoms were secondary to underlying concerns about her mother's history of death from a brain tumor at a similar age and issues related to menopause. To assess her symptoms of fatigue further, a complete blood count, comprehensive chemistry profile, and thyroid-stimulating hormone (TSH) measurement were ordered, all of which were within normal limits with the exception of a mildly decreased glucose level of $57 \mathrm{mg} / \mathrm{dL}$. Her sodium at this time was $135 \mathrm{mEq} / \mathrm{L}$ (normal, 135 to $153 \mathrm{mEq} / \mathrm{L}$ ). Because of her persistent headaches and her concerns regarding her family history of brain tumor, a magnetic resonance image (MRI) of the brain was ordered and was normal.

The patient continued to feel tired during the ensuing 6 weeks despite the reassurance of negative findings on the MRI and despite starting hormone replacement therapy. Depression was considered as a cause, and paroxetine was prescribed. Additional laboratory studies, including a second complete blood count, comprehensive chemistry profile, and TSH measurement were ordered, which showed a sodium of $132 \mathrm{mEq} / \mathrm{L}$, potassium $4.9 \mathrm{mEq} / \mathrm{L}$, and a glucose of $67 \mathrm{mg} / \mathrm{dL}$. Subsequent laboratory values were urine sodium $147 \mathrm{mEq} / \mathrm{L}$ and a morning cortisol of $1.8 \mu \mathrm{g} / \mathrm{dL}$, suggesting adrenal insufficiency. An adrenocorticotropic hormone (ACTH) stimulation test was ordered to confirm the diagnosis, and the baseline, 30-minute, and 60-minute values were $2.5,2.6$, and $2.4 \mu \mathrm{g} / \mathrm{dL}$, respectively. An ACTH level was measured and was elevated at $988 \mathrm{pg} / \mathrm{mL}$, thus confirming the diagnosis of primary adrenal insufficiency. Cortisone acetate, 25 $\mathrm{mg}$ every morning and $12.5 \mathrm{mg}$ every afternoon, was prescribed, and the patient was given educa-
Table 1. Distinguishing Organic and Functional Causes of Fatiguc.

\begin{tabular}{lcc}
\hline Characteristic & Organic & liunctional \\
\hline Chronic $(>6 \mathrm{mo})$ & - & + \\
Course & P'rogressive & liluctuates \\
Identifiable stressors & - & + \\
Slecp disturbance & $+/-$ & + \\
Depressed mond & Reactive or & Primary \\
& secondary & \\
Activity related & Worsens & Improves \\
Morning symptoms worse & - & + \\
Abnormal physical findings & + & - \\
Associated symptoms & Fewer, & Multiple, \\
& specific & nonspecific \\
Family structure & Supportive & Stressful \\
\hline
\end{tabular}

tional materials about Addison disease. Within 1 week of starting cortisone therapy, the patient's symptoms resolved.

\section{Discussion}

Fatigue is a common reason for patients to visit family physicians, and it is associated with many different disease processes. The differential diagnosis for fatigue is extensive and includes psychiatric, infectious, connective tissue, endocrine, neurologic, oncologic, and cardiopulmonary causes.

By far the most common category of diagnosis in fatigued patients is psychiatric disease, which occurs with $60 \%$ to $80 \%$ or more of cases of chronic fatigue. ${ }^{4,6-8}$ Depression accounts for the great majority of cases with a psychiatric cause. Medical causes account for up to $8 \%$ of cases, and of the remainder of patients who have no identifiable medical or psychiatric cause, $4 \%$ meet the criteria for chronic fatigue syndrome. The remainder of cases are undiagnosed despite investigation. ${ }^{6,7}$

Characteristics of the fatigue, associated symptoms, or physical findings can help in distinguishing organic from functional causes (Table 1 ). $\Lambda \mathrm{s}$ in this patient, physical findings are often absent. For patients with signs and symptoms consistent with a psychiatric cause of fatigue, therapy or referral for therapy would be appropriate. For patients with overlapping symptoms or symptoms consistent with organic causes, further workup would be indicated. A study performed at a referral fatigue clinic concluded that a complete blood count, erythrocyte sedimentation rate, comprehensive chemistry profile, and thyroid function tests are the 
Table 2. Causes of Addison Disease.

\begin{tabular}{ll}
\hline Cause & \multicolumn{1}{c}{ Disease } \\
\hline $\begin{array}{l}\text { Autoimmune } \\
\text { Infectious }\end{array}$ & $\begin{array}{l}\text { Polyglandular autoimmune endocrinopathy } \\
\text { Tuberculosis, fungal infections, human } \\
\text { immunodeficiency virus infection }\end{array}$ \\
$\begin{array}{l}\text { Malignancies } \\
\text { Vascular }\end{array}$ & $\begin{array}{l}\text { Lymphoma, metastases } \\
\text { Other }\end{array}$ \\
$\begin{array}{l}\text { Amyloidosis, sarcoidosis, hemochromatosis, } \\
\text { medications (eg, ketoconazole), } \\
\text { congenital hypoplasia, leukodystrophy }\end{array}$ \\
\hline
\end{tabular}

most appropriate initial screening tests for medical disease, with additional testing as indicated by the history and examination findings. ${ }^{7}$ In the case described, the patient's fatigue had overlapping organic and functional signs and symptoms. For example, the fatigue was chronic and progressive, increased with activity, seemed to cause her to feel depressed, but she also had a poor home life, was experiencing apparent menopause, and had no abnormal physical findings. Because of the overlapping organic and functional characteristics of this patient's fatigue, further evaluation was undertaken, which ultimately led to her diagnosis.

Addison disease, or primary adrenal insufficiency, is an uncommon but potentially life-threatening, treatable cause of fatigue. Addison disease is caused by destruction of adrenal cortical tissue generally by immune, infectious, malignant, or vascular mechanisms. In developed countries, Addison disease is caused by autoimmune disease $70 \%$ to $80 \%$ of the time. ${ }^{3,9}$ In these patients, autoantibodies are formed to adrenocortical antigens, and immune destruction with eventual glandular fibrosis and hypofunction occurs. The factors leading to formation of autoantibodies have not been fully determined; however, genetic predisposition is thought to be an important factor. The other major cause of adrenal insufficiency is tuberculosis, which can account for $20 \%$ of cases and might be the leading cause of Addison disease worldwide. Skin testing to screen for tuberculosis should be considered in these patients. Other causes of Addison disease are very uncommon (Table 2 ).

\section{Diagnosis of Addison Disease}

As previously noted, a dominant symptom of Addison disease that occurs in all patients is fatigue. ${ }^{3}$ Anorexia, gastrointestinal complaints, dizziness, salt craving, depression, and menstrual changes can
Table 3. Common Physical and Laboratory Changes in Addison Disease.

Orthostatic blood pressure changes

Weight loss

Hyperpigmentation

Alopecia

Viriligo

Hyponatremia

Hyperkalemia

Anemia, normochromic, normocytic

Hypoglycemia

Hypercalcemia

Azotemia

Eosinophilia

also be found in patients with Addison disease. In addition, depression can occur in $60 \%$ to $80 \%$ of these patients. ${ }^{10}$ Common physical signs and laboratory abnormalities in Addison disease are presented in Table 3. The most frequent laboratory abnormalities are hyponatremia and hyperkalemia, which are found in $90 \%$ and $65 \%$ of patients, respectively. ${ }^{9}$ In the patient described, the finding of hyponatremia led to further evaluation and the diagnosis of adrenal insufficiency. Retrospectively, her other findings (low-normal blood pressure, hypoglycemia, previous low normal sodium levels, and amenorrhea) also suggested and supported this diagnosis.

As this case illustrated, diagnosing Addison disease can be challenging because patients often complain of nonspecific symptoms and have subtle physical findings. Laboratory findings might suggest the need for further investigation (Table 3). Because the severity of illness can vary according to whether the disease is acute or chronic, the steps taken to diagnose Addison disease will depend upon the patient's condition. If the patient is stable, an ACTH stimulation test should be performed by administering $250 \mu \mathrm{g}$ of ACTH after measuring a baseline cortisol level, and then measuring the cortisol levels at 30 and 60 minutes. A normal response would be a value greater than $20 \mu \mathrm{g} / \mathrm{dL}$. For unstable patients with suspected Addison disease, a plasma cortisol should be measured and treatment initiated immediately with intravenous hydrocortisone $100 \mathrm{mg}$, every 6 hours. A rapid ACTH stimulation test, measuring the cortisol level after 30 minutes, could also be performed to expedite diagnosis. ${ }^{3}$ When the diagnosis or cause of adrenal insufficiency is uncertain, plasma ACTH and aldo- 
sterone level measurements, a serum 21 hydroxylase antibody titer determination, and a computerized tomographic scan of the adrenal glands can aid with diagnosis and treatment. In the case discussed, further testing was not performed because of her history of autoimmune thyroid disease and, as discussed below, its known association with other autoimmune endocrine disorders.

A history of an autoimmune disease should heighten one's suspicion of associated autoimmune causes for the patient's symptoms. The patient described in this case had Schmidt syndrome, a polyglandular autoimmune endocrinopathy that occurs in approximately $50 \%$ of patients with Addison disease. ${ }^{3}$ All patients with Schmidt syndrome have adrenal insufficiency. Schmidt syndrome is the association of autoimmune adrenal insufficiency with autoimmune disease in other endocrine organs and, if necessary, can be confirmed with antibody testing. Seventy percent of patients with Schmidt syndrome have evidence of autoimmune thyroid disease $(50 \%$ have Hashimoto thyroiditis, $50 \%$ have Grave disease) and $50 \%$ will have diabetes mellitus. ${ }^{1}$

In $40 \%$ to $50 \%$ of these patients, adrenal insufficiency is the initial diagnosis, and diabetes or thyroid disease is diagnosed later. In the remaining $50 \%$ adrenal insufficiency is found simultaneously or after a diagnosis of thyroid disease or diabetes has been made. Gonadal failure can also occur, and if the patient is perimenopausal, as in this patient, the exact cause of the amenorrhea might be unclear until after the adrenal insufficiency has been treated and stabilized. Onset occurs more commonly in patients aged between 20 and 40 years with a female to male ratio of $1.8: 1$.

Diagnosing Schmidt syndrome involves the same steps and considerations as mentioned above for Addison disease. Screening patients with adrenal insufficiency for thyroid disease can be beneficial; however, thyroid hormone replacement for hypothyroidism should not be prescribed until the patient's condition has stabilized on cortisone replacement. Once stabilized, thyroid testing should be repeated, because in some patients thyroid hormone levels will return to normal.

\section{Treatment}

Daily treatment for adrenal insufficiency involves replacement with oral cortisone acetate, $25 \mathrm{mg} \mathrm{ev}$ ery morning and $12.5 \mathrm{mg}$ every afternoon. Some patients will also require fludrocortisone 0.1 to 0.3 $\mathrm{mg} / \mathrm{d}$. Patient education is a vital part of the treatment. Patients should be instructed to double their dose of cortisone with illness, including upper respiratory tract infections, and to notify their physician when they are ill. The patient who is vomiting will require hospitalization for intravenous therapy. Should patients require surgery, they will need intravenous hydrocortisone replacement until they are tolerating oral intake. Patients should obtain and wear a medical alert bracelet identifying them as having Addison disease.

\section{Conclusion}

Fatigue, a common complaint often attributed to psychiatric disease, can have a variety of medical causes, most of which are uncommon and some of which are potentially life-threatening. Among the medical causes for fatigue is Addison disease. As shown in this case, patients with Addison disease can have fatigue as their primary complaint and have other disorders diagnosed before the diagnosis of adrenal insufficiency is entertained. Although universal laboratory screening of fatigued patients for medical disease is not recommended, findings from assessing the characteristics of the fatigue, reviewing the medical history for potentially causative or related disease, and a physical examination might suggest an organic cause and the need for laboratory screening. Electrolyte abnormalities are the most common findings that will lead to further work-up toward the diagnosis of adrenal insufficiency. In addition, reviewing the chart for previous complaints and physical examination or laboratory findings can be helpful. Previous borderline or mildly abnormal findings (eg, blood pressures, glucose and sodium levels) might provide important clues to the cause of the current complaints and findings and lend further support to the work-up and diagnosis of adrenal insufficiency.

\section{References}

1. Leshin M. Polyglandular autoimmune syndromes. Am J Med Sci 1985;290:77-88.

2. Willis AC, Vince FP. The prevalence of Addison's disease in Coventry, UK. Postgrad Med J 1997;73: 286-8.

3. Miller WL, Tyrell JB. The adrenal cortex. In: Felig P, Baxter JD, Frohman LA, Broadus AE, editors. 
Endocrinology and metabolism. $3^{\text {rd }}$ ed. New York; McGraw-Hill, 1995:642-59.

4. Kroenke K, Wood DR, Mangelsdorff AD, Meier NJ, Powell JB. Chronic fatigue in primary care. Prevalence, patient characteristics, and outcome. JAMA 1988;260:929-34.

5. Buchwald D, Umali P, Umali J, Kith P, Pearlman T, Komaroff AL. Chronic fatigue and the chronic fatigue syndrome: prevalence in a Pacific Northwest health care system. Ann Intern Med 1995;123:81-8.

6. Llewelyn MB. Assessing the fatigued patient. Br J Hosp Med 1996;55:125-9.

7. Lane TJ, Matthews DA, Manu P. The low yield of physical examinations and laboratory investigations of patients with chronic fatigue. Am J Med Sci 1990; 299:313-8.

8. Wessely S, Chalder T, Hirsch S, Wallace P, Wright D. Psychological symptoms, somatic symptoms, and psychiatric disorder in chronic fatigue and chronic fatigue syndrome: a prospective study in the primary care setting. Am J Psychiatry 1996;153:1050-9.

9. Loriaux DL. Adrenocortical insufficiency. In: Becker KL,Bilezikian JP, editors. Principles and practice of endocrinology and metabolism. $2^{\text {nd }}$ ed. Philadelphia: Lippincott-Raven, 1995:682-6.

10. Johnstone PA, Rundell JR, Esposito M. Mental status changes of Addison's disease. Psychosomatics $1990 ; 31: 103-7$. 